\title{
Compact subgroups in the centralizer of natural factors of an ergodic group extension of a rotation determine all factors
}

\author{
MARIUSZ LEMAŃCZYK AND MIECZYSŁAW K MENTZEN \\ Institute of Mathematıcs, Nicholas Copernicus University, ul Chopına 12/18, \\ 87-100 Toruń, Poland
}

(Recelved 27 September 1988)

\begin{abstract}
For ergodic group extensions of transformations with discrete spectra it is proved that each invariant sub- $\sigma$-algebra is determined by a compact subgroup in the centralizer of a natural factor
\end{abstract}

\section{Introduction}

In [5] the set of ergodic measures for compact abehian group extensions of a given transformation was described In the present paper, in a sense, we go further and we study the set of ergodic self-joinings of ergodic group extensions of transformations with discrete spectra These joinıngs turn out to be natural, namely, every ergodic self-joinıng of an ergodic compact, abelian group extension $T_{\varphi}(X \times G, \tilde{\mu}) \rightarrow$ $(X \times G, \tilde{\mu})$ of a transformation with a discrete spectrum $T(X, \mu) \rightarrow(X, \mu)$ must be the relatively independent extension of an isomorphism between some two natural factors of $T_{\varphi}$ (by a natural factor of a $G$-extension $T_{\varphi}$ we mean the action of $T_{\varphi}$ on the quotient space $X \times G / H$, for $H$ a closed subgroup of $G$ )

In [11] (see also [3], [4]) Veech proved that for any ergodic transformation $U$ with the 2-fold simplicity property (we use the definition of 2-fold simplicity from [4]), there was a one-te is correspondence between invariant sub- $\sigma$-algebras and compact subgroups in the centralizer $C(U)$ of $U$ This Veech correspondence is given by

$$
\mathscr{G} \leftrightarrow H(\mathscr{C})=\left\{S \in C(U)(\forall A \in \mathscr{C}) S^{-1} A=A\right\}
$$

for each $U$-invariant sub- $\sigma$-algebra $\mathscr{C}$

In this paper, using the structure of self-joinıngs, we prove that for any $T_{\varphi}$-invariant sub- $\sigma$-algebra of an ergodic group extension of a rotation there is a compact subgroup in the centralizer of a natural factor giving rise to the Veech correspondence

1 Ergodic joinings of group extensions of transformations with discrete spectra Let $T_{1}(l=1, \quad, n)$ be ergodic automorphisms of Lebesgue spaces $\left(X_{1}, \mathscr{B}_{1}, \mu_{1}\right)$, where $\mu_{1}$ is a $T_{1}$-invariant probability measure on a $\sigma$-algebra $\mathscr{B}_{1}$ of subsets of $X_{1}$ 
Definition 1 $[9,4]$ By an n-joining of $T_{1}, \quad, T_{n}$ we mean any $T_{1} \times \quad \times T_{n}$-1nvariant measure $\lambda$ on $\mathscr{B}_{1} \otimes \quad \otimes \mathscr{B}_{n}$ such that for each $\imath=1, \quad, n$ and each $A_{1} \in \mathscr{B}_{1}$

$$
\lambda\left(X_{1} \times \quad \times X_{t-1} \times A_{t} \times X_{t+1} \times \quad \times X_{n}\right)=\mu_{t}\left(A_{t}\right)
$$

The set of all $n$-joinings of $T_{1}, \quad, T_{n}$ will be denoted by $J\left(T_{1}, \quad, T_{n}\right)$ The subset of $J\left(T_{1}, \quad, T_{n}\right)$ consisting of all ergodic measures will be denoted by $J^{e}\left(T_{1}, \quad, T_{n}\right)$ It is clear that if $\lambda \in J\left(T_{1}, \quad, T_{n}\right)$ and

$$
\lambda=\int_{E\left(T_{1} T_{n}\right)} e d \tau(e)
$$

1s its ergodic decomposition with $E\left(T_{1}, \quad, T_{n}\right)$ being the set of all ergodic measures on $\mathscr{B}_{1} \otimes \otimes \mathscr{B}_{n}$, then

$$
\tau\left(J^{e}\left(T_{1}, \quad, T_{n}\right)\right)=1
$$

Hence, we can say that the ergodic components of an $n$-joining are $n$-joinings In particular, $J^{e}\left(T_{1}, \quad, T_{n}\right)$ is nonempty since $\mu_{1} \times \cdot \quad \times \mu_{n} \in J\left(T_{1}, \quad, T_{n}\right)$

If $n=2$ we say (for short) joinings (1nstead of 2-joinıngs) If $T_{1}=T_{2}=\quad=T_{n}=T$ we say $n$-self-joinings of $T$

Let $T(X, \mathscr{B}, \mu) \rightarrow(X, \mathscr{B}, \mu)$ be an ergodic automorphism By the centralizer, $C(T)$, of $T$ we mean the set of all $S(X, \mathscr{B}, \mu) \rightarrow(X, \mathscr{B}, \mu)$ commuting with $T, 1 \mathrm{e}$ $S T=T S$ This set is endowed with the weak topology given by $S_{n} \rightarrow S$ iff for each $A \in \mathscr{B}, \mu\left(S_{n}^{-1} A \Delta S^{-1} A\right) \rightarrow 0$ For any $S \in C(T)$ we can define the corresponding graph joining $\mu_{S}$ defined on rectangles as

$$
\mu_{S}(A \times B)=\mu\left(A \cap S^{-1} B\right)
$$

The following characterization of graph joinıngs can be easily proved

LEMMA 1 Let $\lambda \in J^{e}(T, T)$ Then $\lambda$ is a graph joining iff for any $A \in \mathscr{B}$ there is $\mathscr{B} \in \mathscr{B}$ with $\lambda(A \times X \Delta X \times B)=0$, te $\lambda$ tdentifies the two marginals sub- $\sigma$-algebras of $\mathscr{B} \otimes \mathscr{B}$

If $S_{1}, \quad, S_{n-1} \in C(T)$ then the measure defined as

$$
\mu_{S_{1}}, S_{n-1}\left(A_{0} \times A_{1} \times \quad \times A_{n-1}\right)=\mu\left(A_{0} \cap S_{1}^{-1} A_{1} \cap \quad \cap S_{n-1}^{-1} A_{n-1}\right)
$$

is an element of

$$
J^{e}(\underbrace{T, \quad, T)}_{n \times}
$$

Any $T$-invariant sub- $\sigma$-algebra $\ell \subset \mathscr{B}$ is called a factor of $T$ (more precisely, the action of $T$ on $\ell$ is called a factor of $T$ on $\mathscr{B}$ ) Assume, that two factors $\ell_{1}, \ell_{2}$ are 1somorphic, 1 e there exists

$$
S\left(T, X_{1}, \ell_{1}, \mu\right) \rightarrow\left(T, X_{2}, \ell_{2}, \mu\right),
$$

where $X_{1}, X_{2}$ are the corresponding quotients We can lift this isomorphism to a self-joining $\lambda$ of $T$ by

$$
\lambda(A \times B)=\int_{X_{1}} E\left(A \mid \ell_{1}\right)(\bar{x}) E\left(B \mid \ell_{2}\right)(S \bar{x}) d \mu(\bar{x})
$$


Such a joining is called the relatively independent extension of the isomorphism $S$ Note that $\lambda$ need not be ergodic. In particular, when $\ell=\ell_{1}=\ell_{2}$ and $S=1 \mathrm{~d}, \lambda$ is called the relatively independent extension of the diagonal measure on $\ell$ This joining also need not be ergodic

From now on we assume that $T(X, \mathscr{B}, \mu) \rightarrow(X, \mathscr{B}, \mu)$ is an ergodic transformation with discrete spectrum, i e $L^{2}(X, \mathscr{B}, \mu)=\operatorname{span}\left\{f_{\alpha} \alpha \in \operatorname{Sp}(T), f_{\alpha} \circ T=\alpha f_{\alpha}\right\}$, where $S p(T)$ is the point spectrum of the unitary operator $T L^{2}(X, \mathscr{B}, \mu) \rightarrow$ $L^{2}(X, \mathscr{B}, \mu), T f=f \circ T$

Proposition 1 Let $T \cdot(X, \mathscr{B}, \mu) \rightarrow(X, \mathscr{B}, \mu)$ be an ergodic transformation with discrete spectrum Then

(1) $C(T)$ is a group, and

(11) $J^{e}(T, T)=\left\{\mu_{S} \quad S \in C(T)\right\}$

Proof Obviously (1) follows from (11) Take $\lambda \in J^{e}(T, T)$ We will show that $\lambda$ identıfies two marginal sub- $\sigma$-algebras $\tilde{\mathscr{B}}_{1}=\{A \times X \quad A \in \mathscr{B}\}, \tilde{\mathscr{B}}_{2}=\{X \times B \quad B \in \mathscr{B}\}$ To this end let us look at $L^{2}(X \times X, \mathscr{B} \otimes \mathscr{B}, \lambda)$ and the corresponding marginal subspaces

$$
\begin{aligned}
& L^{2}\left(\tilde{\mathscr{B}}_{1}\right)=\left\{\tilde{f} \tilde{f}(x, y)=f(x), f \in L^{2}(X, \mu)\right\}, \\
& L^{2}\left(\tilde{\mathscr{B}}_{2}\right)=\left\{\tilde{\tilde{f}} \tilde{f}(x, y)=f(y), f \in L^{2}(X, \mu)\right\}
\end{aligned}
$$

Since $\lambda \in J(T, T)$, both $L^{2}\left(\tilde{\mathscr{B}}_{1}\right)$ and $L^{2}\left(\tilde{\mathscr{B}}_{2}\right)$ are naturally identified with $L^{2}(X, \mu)$ Therefore they are spanned by $\left\{\tilde{f}_{\alpha} \alpha \in \operatorname{Sp}(T)\right\},\left\{\tilde{\tilde{f}}_{\alpha} \quad \alpha \in \mathrm{Sp}(T)\right\}$ respectively But $\lambda$ is ergodic, so

$$
\tilde{f}_{\alpha}=a_{\alpha} \quad \tilde{f}_{\alpha}, \quad a_{\alpha} \in \mathbb{C}
$$

and consequently $L^{2}\left(\tilde{\mathscr{B}}_{1}\right)=L^{2}\left(\tilde{\mathscr{B}}_{2}\right)$ as two subspaces in $L^{2}(X \times X, \lambda)$ This is equivalent to sayıng that $\tilde{\mathscr{B}}_{1}$ and $\tilde{\mathscr{B}}_{2}$ are identıfied by $\lambda$ An application of Lemma 1 gives the result

Remark The notion of graph joinıng (1) can be easily transferred to the case $J^{e}\left(T_{1}, T_{2}\right)$ where we consider isomorphism between $T_{1}$ and $T_{2}$. Lemma 1 still works and the proof of Proposition 1 gives rise to a new proof of the well-known result that if $T_{1}$ and $T_{2}$ are ergodic transformations with discrete spectrum and $\mathrm{Sp}\left(T_{1}\right)=\mathrm{Sp}\left(T_{2}\right)$ then they are isomorphic (actually each ergodic joinıng between $T_{1}$ and $T_{2}$ is the graph of an isomorphism)

As an immediate consequence of Proposition 1 we get

COROLlaRy 1 If $T(X, \mathscr{B}, \mu) \rightarrow(X, \mathscr{B}, \mu)$ is an ergodic automorphism with discrete spectrum then

$$
J^{e}(\underbrace{T, \quad, T)}_{n}=\left\{\mu_{S_{1}} \quad s_{n-1} S_{1}, \quad, S_{n-1} \in C(T)\right\} .
$$

Let $G$ be a compact metric abelian group equipped with a normalized Haar measure $\nu$ Let $\varphi \quad X \rightarrow G$ be a measurable map Define

$$
\begin{gathered}
T_{\varphi}(X \times G, \mu \times \nu) \rightarrow(X \times G, \mu \times \nu), \\
T_{\varphi}(x, g)=(T X, \varphi(x) g)
\end{gathered}
$$


$T_{\varphi}$ is called a group extension of $T$ Following [8] $T_{\varphi}$ is ergodic iff whenever $\alpha \in \hat{G}$ (1 e $\alpha$ is a character of $G$ ) and a measurable

$$
h X \rightarrow S^{1}=\{z \in \mathbb{C}|z|=1\} \text { satisfy } h(T x) h(x)^{-1}=\alpha(\varphi(x)),
$$

then $\alpha=1$

We will also use the following result

Proposition 2 [7] Let $T_{\varphi}$ be an ergodic G-extension of $T$ Let $\bar{S} \in C\left(T_{\varphi}\right)$ Then there are a contınuous group epımorphism $v G \rightarrow G$ a measurable map $f X \rightarrow G$ and $S \in C(T)$ such that

$$
\bar{S}(x, y)=S_{f, v}(x, y)=(S x, f(x) v(y))
$$

Let $H \subset G$ be a closed (compact) subgroup Then we can consider the action of $T_{\varphi}$ on $X \times G / H$ The factors of this form are called natural factors In fact these are the only factors of $T_{\varphi}$ that contain the $\sigma$-algebra $\{A \times G A \in \mathscr{B}\}([4],[11])$

Our aim is to describe all ergodic self-joinings for an ergodic $G$-extension of $T_{\varphi}$ Without loss of generality we can assume that $T$ is an ergodic rotation on a compact monothetic group $X$ First we will work with the situation where $T_{\varphi}$ is not necessarily ergodic Take an ergodic component $\lambda$

Let $\Pi X \times G \rightarrow X, \Pi(x, g)=x$ Then, if $\lambda$ is an ergodic $T_{\varphi}$-invariant measure on $X \times G$ then $\lambda \Pi^{-1}$ is $T$-ergodic, hence $\lambda \Pi^{-1}=\mu$ We will also use the following straightforward result

LEMMA 2 There is a measurable $T_{\varphi}$-invariant subset $Y \subset X \times G, \lambda(Y)=1$ such that for each $(x, g) \in Y$ and for each continuous function $f$ on $X \times G$ ( i e $f \in C(X \times G)$ )

$$
\lim _{n \rightarrow \infty} S_{n}(f)(x, g) \lim _{n \rightarrow \infty} \frac{1}{n} \sum_{k=0}^{n-1} f \circ\left(T_{\varphi}\right)^{k}(x, g)=\int f d \lambda
$$

Let us denote by $H$ the stabilizer of $\lambda$ in $G, 1 \mathrm{e}$

$$
H=\{g \in G \quad \lambda g=\lambda\},
$$

where $\lambda g(A \times B)=\lambda\left(A \times B g^{-1}\right)$ or

$$
\int f(x, g) d(\lambda g)=\int f(x, h g) d \lambda \quad \text { for } f \in C(X \times G)
$$

Let us denote $f \circ g(x, h)=f(x, h g)$

LEMMA 3 (1) $H$ is a closed subgroup of $G(11)$ If $(x, g),(x, h) \in y$ then $h H=g H$

Proof As (1) is obvious, we will prove (11) Take $f \in C(X \times G)$ Then $(x, g) \in Y$ implies $S_{n}(f)(x, g) \rightarrow_{n} \int f d \lambda$ But

$$
S_{n}(f)(x, g)=S_{n}(f)\left(x, h h^{-1} g\right)=S_{n}\left(f \circ h^{-1} g\right)(x, h)
$$

since the action of $G$ on the second coordinate commutes with $T_{\varphi}$ But from our assumption, $(x, h) \in Y$ so

$$
S_{n}\left(f \circ h^{-1} g\right)(x, h) \underset{n \rightarrow x}{\longrightarrow} \int f \circ h^{-1} g d \lambda=\int f d\left(\lambda h^{-1} g\right)
$$

Because $f$ is an arbitrary element of $C(X \times G), \lambda h^{-1} g=\lambda$, or, similarly, $h^{-1} g \in H$ 
Let us decompose $\lambda$ over the factor $(X, T, \mu)$

$$
\lambda=\int_{X} \lambda_{x} d \mu(x)
$$

LEMMA $4 \lambda_{x}=\nu_{H} g \mu$-a e, where $\nu_{H}$ is Haar measure on $H, g=g(x)$ and $(x, g) \in Y$ Proof Let $A$ be a Borel subset of $G, h \in H$ Let

$$
M=\left\{x \in X \quad \lambda_{x}\left(A h^{-1}\right)<\lambda_{x}(A)\right\}
$$

and suppose that $\mu(M)>0$ Then

$$
\begin{aligned}
\lambda(M \times A) & =\lambda h(M \times A)=\lambda\left(M \times A h^{-1}\right)=\int_{M} \lambda_{x}\left(A h^{-1}\right) d \mu(x) \\
& <\int_{M} \lambda_{x}(A) d \mu(x)=\lambda(M \times A),
\end{aligned}
$$

a contradiction Similarly we show that $\mu\left\{x \in X \quad \lambda_{x}\left(A h^{-1}\right)>\lambda_{x}(A)\right\}=0$ As a conclusion we have $\lambda_{x} h=\lambda_{x} \mu$-a e Let $(x, g) \in Y$ From Lemma 3(11) it follows that

$$
Y \cap(\{x\} \times G)=\{x\} \times g H
$$

Hence $\lambda_{x}(g H)=1$ This implies $\lambda_{x} g^{-1}(H)=1$ But for $h \in H$

$$
\left(\lambda_{x} g^{-1}\right) h=\left(\lambda_{x} h\right) g^{-1}=\lambda_{x} g^{-1}
$$

Thus $\lambda_{x} g^{-1}$ is invariant under all translations by elements of $H$ and therefore $\lambda_{x} g^{-1}=\nu_{H}$

Remark Lemma 4 implies that if we denote by $\tilde{\lambda}=\int_{X} \tilde{\lambda}_{x} d \mu(x)$ the image of $\lambda$ on $X \times G / H$ then $\tilde{\lambda}_{x}$ is a Dirac measure $\mu$-a e This allows us to define a measurable function $f X \rightarrow G / H$ by

$$
f(x)=\left(\tilde{\lambda_{x}}\right)^{-1}(1)
$$

$1 \mathrm{e} f(x)$ is the only atom of $\tilde{\lambda}_{x}$ on $G / H$ ( $f$ is measurable since $f^{-1}(A)=$ $E\left(\chi X \times A_{X \times A} \mid \mathscr{B}\right)^{-1}(1)$, for any Borel subset $\left.A \subset G / H\right)$ Moreover, the $T_{\varphi}$-1nvariance of $\lambda$ implies

$$
f(T x)=\varphi(x) f(x)
$$

LEMMA 5 The system $\left(X \times G, T_{\varphi}, \lambda\right)$ is isomorphic to $\left(X \times H, T_{\psi}, \mu \times \nu_{H}\right)$ where $\psi X \rightarrow H$ is measurable (ie ergodic $T_{\varphi}$-invariant measures induce ergodic group extensions automorphisms)

Proof Define $t \quad X \rightarrow G$ by the formula $t(x)=U(f(x))$, where $U$ is a measurable selector for the natural map $G \rightarrow G / H$ (see [10], p 5), 1 e $U$ satisfies $U(g H) H=g H$ Then $t(x) \in f(x) \mu$-a e and, by $(6), \varphi(x) t(x) H=t(T x) H$ Put

$$
\psi(x)=\varphi(x) t(T x)^{-1} t(x) \in H
$$

Therefore from Lemma 4 it follows that

$$
J\left(X \times H, T_{\psi}, \mu \times \nu_{H}\right) \rightarrow\left(X \times G, T_{\varphi}, \lambda\right)
$$

acting as $J(x, h)=(x, t(x) h)$ is an isomorphism

Let

$$
\begin{gathered}
\Gamma=\left\{\gamma \in \hat{G} \text { there is a measurable } h \quad X \rightarrow S^{1}\right. \text { such that } \\
\left.h(T x) h(x)^{-1}=\gamma(\varphi(x)) \mu \text {-a e }\right\}
\end{gathered}
$$


Then $\Gamma$ is a subgroup of $\hat{G}$ and put

$$
F=\operatorname{ann} \Gamma=\{g \in G \text { for each } \gamma \in \Gamma, \gamma(g)=1\}
$$

LEMMA $6 F=H$

Proof Let $g_{0} \in H, \gamma \in \Gamma$ Then $\gamma(\varphi(x))=h(T x) h(x)^{-1}$ and let us define a function w $X \times G \rightarrow S^{1}$ setting

$$
w(x, g)=h(x)^{-1} \gamma(g)
$$

Then for $\mu$-a e $x$ and for all $g, w$ is $T_{\varphi}$-invariant The ergodicity of $\lambda$ forces $w$ to be constant $\lambda$-a.e, 1 e $h(x)^{-1} \gamma(g)=c \neq 0$ Moreover

$$
\begin{aligned}
c & =\int w(x, g) d \ell=\int h(x)^{-1} \gamma(g) d \lambda=\int h(x)^{-1} \gamma(g) d\left(\lambda g_{0}\right) \\
& =\int h(x)^{-1} \gamma\left(g g_{0}\right) d \lambda=\gamma\left(g_{0}\right) c
\end{aligned}
$$

Hence $\gamma\left(g_{0}\right)=1$ and therefore $g_{0} \in F$ Now, let $g \in F$ If $g \notin H$ then there is a character $\gamma$ such that

$$
\gamma(g) \neq 1 \text { and } \quad \gamma(H)=1
$$

From (7) it follows that

$$
\gamma(\varphi(x))=\gamma\left(t(T x) \psi(x) t(x)^{-1}\right)=\gamma(t(T x)) \gamma(t(x))^{-1},
$$

since $\psi(x) \in H$ This implies $\gamma \in \Gamma$ and consequently $\gamma(g)=1$ which is a contradiction Remark. The results contained in Lemmata 3-6 can be deduced from [5] We include these results for completeness as well as for new and simple proofs

Now, we are in a position to pass to our main problem, namely, to describe all ergodic self-joinıngs of $T_{\varphi}$ We assume that

$$
T_{\varphi} \text { is an ergodic G-extension }
$$

Let $\Pi X \times G \times X \times G \rightarrow X \times X$ be defined as

$$
\Pi(x, g, y, h)=(x, y)
$$

Assume that $\bar{\lambda} \in J^{e}\left(T_{\varphi}, T_{\varphi}\right)$ Then by Proposition 1,

$$
\bar{\lambda} \Pi^{-1}=\mu_{S}
$$

for some $S \in C(T)$ Hence

LEMMA 7

$$
\bar{\lambda}\left(\bigcup_{x \in X}\{x\} \times G \times\{S x\} \times G\right)=1
$$

We define a measure $\lambda$ on $X \times G \times G$ as follows

$$
\lambda(A \times B \times C)=\bar{\lambda}(A \times B \times S A \times C)
$$

Put

$$
\begin{gathered}
\alpha \bigcup_{x \in X}\{x\} \times G \times\{S x\} \times G \rightarrow X \times G \times G, \\
\alpha(x, g, S x, h)=(x, g, h)
\end{gathered}
$$


Then we see that $\lambda$ is just the image of $\bar{\lambda}$ via $\alpha$ Also $T_{\varphi \times \varphi \circ S} \circ \alpha=\alpha \circ\left(T_{\varphi} \times T_{\varphi}\right)$ Therefore the Lemma below is clear

LEMMA 8 The function $\alpha$ is an isomorphism of $\left(X \times G \times X \times G, T_{\varphi} \times T_{\varphi}, \bar{\lambda}\right)$ and $\left(X \times G \times G, T_{\varphi \times \varphi \circ s}, \lambda\right)$

In what follows we will consider $T_{\varphi \times \varphi \circ S}$ and the measure $\lambda$ on $X \times G \times G$ Let $H \subset G \times G, H=\left\{\left(g_{1}, g_{2}\right) \in G \times G \lambda\left(g_{1}, g_{2}\right)=\lambda\right\}$,

$$
H_{1}, H_{2} \subset G, \quad H_{1}=\{g \in G(g, e) \in H\}, \quad H_{2}=\{g \in G(e, g) \in H\},
$$

where $e$ is the unit element of $G$

Then, obviously, $H_{1}, H_{2}$ are closed subgroups of $H$ If we put $\Gamma=$ $\left\{\left(\gamma_{1}, \gamma_{2}\right) \in \hat{G} \times \hat{G}\right.$ there is a measurable function $h X \rightarrow S^{1}$ such that $\gamma_{1}(\varphi(x)) \gamma_{2}(\varphi(S x))=h(T x) h(x)^{-1}$ then from Lemma $6, H=$ ann $\Gamma$ and therefore

$$
H_{1}=\operatorname{ann} \Gamma_{l}, \quad l=1,2,
$$

where $\Gamma_{t}=\Pi_{i}(\Gamma), \Pi_{1} \hat{G} \times \hat{G} \rightarrow \hat{G}, \Pi_{l}\left(\gamma_{1}, \gamma_{2}\right)=\gamma_{1}$

LEMMA $9 \Gamma$ is a 'diagonal' subgroup of $\hat{G} \times \hat{G}, \imath e$,

$$
\begin{array}{ll}
\left(\gamma_{1}, \gamma_{2}\right) \in \Gamma,\left(\gamma_{1}, \gamma_{2}^{\prime}\right) \in \Gamma \quad \text { imply } \gamma_{2}=\gamma_{2}^{\prime}, \\
\left(\gamma_{1}, \gamma_{2}\right) \in \Gamma,\left(\gamma_{1}^{\prime}, \gamma_{2}\right) \in \Gamma \quad \text { imply } \gamma_{1}=\gamma_{1}^{\prime}
\end{array}
$$

Proof This is an obvious consequence of ergodicity of $T_{\varphi}$ and (3)

LEMMA 10 There is a group isomorphism $\hat{w} \quad \Gamma_{2} \rightarrow \Gamma_{1}$

Proof This follows from Lemma 9 (as $\Gamma$ is a subgroup of $\hat{G} \times \hat{G}$ ) that

$$
\hat{w}\left(\gamma_{2}\right)=\gamma_{1} \quad \text { iff } \quad\left(\gamma_{1}, \gamma_{2}\right) \in \Gamma
$$

1s a well-defined group 1somorphism of $\Gamma_{1}$ and $\Gamma_{2}$

Let $w G / H_{1} \rightarrow G / H_{2}$ be the group isomorphism determined by

$$
\begin{aligned}
& \hat{w}\left(G / H_{2}\right) \hat{\rightarrow}\left(G / H_{1}\right), \\
& \hat{w}\left(\gamma_{2}\right)=\gamma_{2} w,
\end{aligned}
$$

where $\left(G / H_{t}\right)^{\wedge}$ is naturally identified with $\Gamma_{\text {, }}$ as ann $\Gamma_{1}=H_{\imath}, \imath=1,2$

LEMMA 11

$$
H=\bigcup_{g \in G} g H_{1} \times w\left(g^{-1} H_{1}\right)
$$

Proof Let $g \in G,\left(\hat{w}\left(\gamma_{2}\right), \gamma_{2}\right) \in \Gamma$ Then

$$
\begin{aligned}
\left(\hat{w}\left(\gamma_{2}\right), \gamma_{2}\right)\left(g H_{1} \times w\left(g^{-1} H_{1}\right)\right) & =\hat{w}\left(\gamma_{2}\right)\left(g H_{1}\right) \gamma_{2}\left(w\left(g^{-1} H_{1}\right)\right) \\
& =\hat{w}\left(\gamma_{2}\right)\left(g H_{1}\right) \quad \hat{w}\left(\gamma_{2}\right)\left(g^{-1} H_{1}\right) \\
& =\hat{w}\left(\gamma_{2}\right)\left(H_{1}\right)=1,
\end{aligned}
$$

since (8) holds Therefore $g H_{1} \times w\left(g^{-1} H_{1}\right) \subset H$

Now, let $(g, h) \in H$ We wish to show that

$$
h H_{2} \quad w\left(g H_{1}\right)=H_{2}
$$


Indeed, let $\gamma_{2} \in \Gamma_{2}$,

$$
\begin{aligned}
\gamma_{2}\left(h H_{2} \quad w\left(g H_{1}\right)\right) & =\gamma_{2}\left(w\left(g H_{1}\right)\right) \quad \gamma_{2}\left(h H_{2}\right)=\hat{w}\left(\gamma_{2}\right)\left(g H_{1}\right) \quad \gamma_{2}\left(h H_{2}\right) \\
& =\left(\hat{w}\left(\gamma_{2}\right), \gamma_{2}\right)\left(g H_{1} \times h H_{2}\right)=1
\end{aligned}
$$

Now, from (9), $w\left(g H_{1}\right)^{-1}=h H_{2}$, so $h \in w\left(g^{-1} H_{1}\right)$

Let $\hat{p} \quad \Gamma_{2} \rightarrow \Gamma$ be defined by

$$
\hat{p}\left(\gamma_{2}\right)=\left(\hat{w}\left(\gamma_{2}\right), \gamma_{2}\right)
$$

and let $p(G \times G) / H \rightarrow G / H_{2}$ be determined by

$$
\hat{p}\left(\gamma_{2}\right)=\gamma_{2}^{\circ} p
$$

Put $\bar{f} X \rightarrow G / H_{2}$

$$
\bar{f}=p \circ f
$$

where $f$ is defined by (5)

Let $v G / H_{1} \rightarrow G / H_{2}$ be the topological group isomorphism defined by

$$
v\left(g H_{1}\right)=w\left(g^{-1} H_{1}\right)
$$

Finally, let us define $S_{\bar{f} v} X \times G / H_{1} \rightarrow X \times G / H_{2}$ setting

$$
S_{\bar{f}, v}\left(x, g H_{1}\right)=\left(S x, \bar{f}(x) v\left(g H_{1}\right)\right)
$$

LEMMA 12 The map $S_{\bar{f}, v}$ establishes an isomorphism of the natural factors $\left(X \times G / H_{1}, T_{\varphi}, \mu \times \nu\right)$ and $\left(X \times G / H_{2}, T_{\varphi}, \mu \times \nu\right)$

Proof It is sufficient to show that $S_{\bar{f}, v} \circ T_{\varphi}=T_{\varphi} \circ S_{\bar{f}, v}$ This is equivalent to proving the equality

$$
\bar{f}(T x) \bar{f}(x)^{-1} v\left(\varphi(x) H_{1}\right) \varphi(S x)^{-1} H_{2}=H_{2} \quad \mu \text {-a e }
$$

Using (6) and (12), (15) can be reduced to showing that

$$
p((\varphi(x), \varphi(S x)) H) v\left(\varphi(x) H_{1}\right) \varphi(S x)^{-1} H_{2}=H_{2}
$$

Take $\gamma \in \Gamma_{2}$ Then by (12), (10) and (13)

$$
\begin{aligned}
& \gamma\left[p((\varphi(x), \varphi(S x)) H) \quad v\left(\varphi(x) H_{1}\right) \varphi(S x)^{-1} H_{2}\right] \\
& =\gamma \circ p((\varphi(x), \varphi(S x)) H) \quad \gamma\left(v\left(\varphi(x) H_{1}\right)\right) \quad \gamma\left(\left(\varphi(S x)^{-1}\right) H_{2}\right) \\
& =\hat{p}(\gamma)((\varphi(x), \varphi(S x)) H) \quad \gamma\left(v\left(\varphi(x) H_{1}\right)\right) \quad \gamma\left(\left(\varphi(S x) H_{2}\right)^{-1}\right) \\
& =\hat{w}(\gamma)\left(\varphi(x) H_{1}\right) \quad \gamma\left(\varphi(S x) H_{2}\right) \quad \gamma\left(v\left(\varphi(x) H_{1}\right)\right) \quad \gamma\left(\varphi(S x) H_{2}\right)^{-1}=1
\end{aligned}
$$

THEOREM 1 If $T_{\varphi}(X \times G, \mu \times \nu) \rightarrow(X \times G, \mu \times \nu)$ is an ergodic group extension of a transformation with discrete spectrum and $\bar{\lambda} \in J^{e}\left(T_{\varphi}, T_{\varphi}\right)$ then there exist closed subgroups $H_{1} \subset G, H_{2} \subset G$ and an isomorphism of the corresponding natural factors $\bar{S}\left(X \times G / H_{1}, T_{\varphi}\right) \rightarrow\left(X \times G / H_{2}, T_{\varphi}\right)$ such that for any Borel sets $A \subset X \times G$, $B \subset X \times G$

$$
\bar{\lambda}(A \times B)=\int_{X \times G / H_{1}} E\left(A \mid H_{1}\right)\left(x, g H_{1}\right) \quad E\left(B \mid H_{2}\right)\left(\bar{S}\left(x, g H_{1}\right)\right) d(\mu \times \nu)\left(x, g H_{1}\right),
$$

where $E\left(A \mid H_{t}\right)$ denotes the conditional expectation with respect to the natural factor $\left(X \times G / H_{\imath}, \mu \times \nu\right), l=1,2$ ( $\iota$ e $\bar{\lambda}$ is the relatively independent extension of an isomorphism of two natural factors) 
Proof It follows from Lemmata 4, 11, 12, since

$$
\nu_{H}=\int_{G / H_{1}} \nu_{H_{1}} g H_{1} \times \nu_{H_{2}} v\left(g H_{1}\right) d \nu\left(g H_{1}\right)
$$

Although we have dealt with the case $\bar{\lambda} \in J^{e}\left(T_{\varphi}, T_{\varphi}\right)$, all Lemmata 7-12 go through when $\bar{\lambda} \in J^{e}\left(T_{\varphi_{1}}, T_{\varphi_{2}}\right)$ where $\varphi_{1} \quad X \rightarrow G_{1}, \varphi_{2} X \rightarrow G_{2}, T_{\varphi_{1}}, T_{\varphi_{2}}$ are ergodic This proves the following

THEOREM 2 Let $T_{\varphi_{1}}\left(X \times G_{i}, \mu \times \nu_{i}\right) \rightarrow\left(X \times G_{i}, \mu \times \nu_{i}\right)$ be an ergodic group extension of $T, \imath=1,2$ If $\bar{\lambda} \in J^{e}\left(T_{\varphi_{1}}, T_{\varphi_{2}}\right)$ then there exist two closed subgroups $H_{\imath} \subset G_{1}$ and an isomorphism of the natural factors

$$
\bar{S}\left(X \times G_{1} / H_{1}, T_{\varphi_{1}}, \mu \times \nu_{1}\right) \rightarrow\left(X \times G_{2} / H_{2}, T_{\varphi_{2}}, \mu \times \nu_{2}\right)
$$

such that for any Borel sets $A \subset X \times G_{1}, B \subset X \times G_{2}$

$$
\bar{\lambda}(A \times B)=\int_{X \times G_{1} / H_{1}} E\left(A \mid H_{1}\right)\left(x, g H_{1}\right) \quad E\left(B \mid H_{2}\right)\left(\bar{S}\left(x, g H_{1}\right)\right) d\left(\mu \times \nu_{1}\right)\left(x, g H_{1}\right)
$$

Remark. A combınation of Lemma 5 and Theorem 2 allows us to describe all ergodic $n$-joinıngs of ergodic extensions $T_{\varphi_{1}}, \quad, T_{\varphi_{n}}$ Indeed, let $\lambda \in J^{e}\left(T_{\varphi_{1}}, \quad, T_{\varphi_{n}}\right)$ Then the measure $\bar{\lambda}$ given by

$$
\bar{\lambda}\left(A_{1} \times A_{2} \times \quad \times A_{n-1}\right)=\lambda\left(A_{1} \times \quad \times A_{n-1} \times\left(X \times G_{n}\right)\right)
$$

is an ergodic $n-1$-joining of $T_{\varphi_{1}}, \quad, T_{\varphi_{n-1}}$ From Lemma 5 it follows that $\left(T_{\varphi_{1}} \times \quad \times T_{\varphi_{n-1}}, \bar{\lambda}\right)$ is isomorphic to some $H$-extension of $T$ Therefore $\lambda$ is an ergodic joining of this $H$-extension of $T$ and $T_{\varphi_{n}}$ Then we apply Theorem 2

Remark The result of Theorem 2 can be generalized as follows Let $T(X, \mathscr{B}, \mu) \rightarrow$ $(X, \mathscr{B}, \mu)$ be an ergodic (not necessarily with a discrete spectrum) transformation of a Lebesgue space, $\varphi \quad X \rightarrow G$ an ergodic cocycle If $\bar{\lambda} \in J^{e}\left(T_{\varphi}, T_{\varphi}\right)$ projected on $J^{e}(T, T)$ is the graph joinıng of an $S \in C(T)$ then $\bar{\lambda}$ must satisfy the conclusion of Theorem 1

\section{Structure of factors of group extensions of transformations with discrete} spectra (Veech theorem)

Let $T_{\varphi}(X \times G, \tilde{\mathscr{B}}, \tilde{\mu}) \rightarrow(X \times G, \tilde{\mathscr{B}}, \tilde{\mu})$ be an ergodic group extension of a transformation with discrete spectrum $T(X, \mathscr{B}, \mu) \rightarrow(X, \mathscr{B}, \mu), \tilde{\mu}=\mu \times \nu_{G}$ and $\tilde{\mathscr{B}}$ the corresponding product $\sigma$-algebra For each closed subgroup $H \subset G$ we have a natural factor $T_{\varphi}(X \times G / H, \tilde{\mu}) \rightarrow(X \times G / H, \tilde{\mu})$. Let $C_{1}\left(T_{\varphi}, H\right)$ denote the group of all invertible elements of the centralizer of $T_{\varphi}$ on $(X \times G / H, \tilde{\mu})$ Assume that $\mathscr{H} \subset C_{1}\left(T_{\varphi}, H\right)$ is a subgroup Then this $\mathscr{H}$ determines a factor of $T_{\varphi}$ on $X \times G / H$ (and hence a factor of $T_{\varphi}$ on $X \times G$ ) by

$$
\ell(\mathscr{H})=\{A \in \tilde{\mathscr{B}} \text { for each } \bar{S} \in \mathscr{H}, \bar{S} A=A\}
$$

The point is that when we pass through all compact $\mathscr{H}$ for all possible closed $H \subset G$ we get all factors (Theorem 3) 
Let $\ell \subset \tilde{B}$ be a $T_{\varphi}$-1nvariant sub- $\sigma$-algebra Following (2) this $\ell$ gives rise to a self-joining of $T_{\varphi}$ by

$$
\tilde{\mu} \times_{\ell} \tilde{\mu}(A \times B)=\int_{\bar{x}} E(A \mid \ell)(\bar{x}) \quad E(B \mid \ell)(\bar{x}) d \tilde{\mu}(\bar{x}),
$$

where $\bar{X}$ is the quotient corresponding to $\ell$

Put $\lambda=\tilde{\mu} \times_{\ell} \tilde{\mu}$ Since $\lambda$ is not necessarily ergodic, let

$$
\lambda=\int_{J^{e}\left(T_{\varphi}, T_{\varphi}\right)} e d \gamma(e)
$$

be its ergodic decomposition, $\gamma$ a probability measure on $J^{e}\left(T_{\varphi}, T_{\varphi}\right)$

The following lemma is well-known ([4], [9])

Lemma 13 Let $A$ be a Borel subset of $X \times G$ Then $A \in \ell$ iff $\lambda\left(A \times A^{c} \cup A^{c} \times A\right)=0$

Let $E=\left\{e \in J^{e}\left(T_{\varphi}, T_{\varphi}\right)\right.$ for each $\left.A \in \ell, e\left(A \times A^{c} \cup A^{c} \times A\right)=0\right\}$

LEMMA $14 \gamma(E)=1$

The proof of this is easy and is therefore omitted

Lemma 15 Let $e \in J^{e}\left(T_{\varphi}, T_{\varphi}\right)$ By Theorem 1 ,

$$
e=\int_{X \times G / H_{1}} E\left(\mid H_{1}\right)\left(x, g H_{1}\right) \cdot E\left(\mid H_{2}\right)\left(S_{f, v}\left(x, g H_{1}\right)\right) d \tilde{\mu}\left(x, g H_{1}\right)
$$

Then $e \in E$ iff $\ell \subset \tilde{\mathscr{B}}_{\mathrm{H}_{1} \mathrm{H}_{2}}$ and for each $A \in \ell, S_{f, v}^{-1}(A)=A$

Proof We start with the following observation

$$
\tilde{\mathscr{B}}_{\mathrm{H}_{1}} \cap \tilde{\mathscr{B}}_{\mathrm{H}_{2}}=\tilde{\mathscr{B}}_{\mathrm{H}_{1} \mathrm{H}_{2}}
$$

since $\tilde{\mathscr{B}}_{J}=\{A \in \tilde{\mathscr{B}}$ for each $g \in J, A g=A\}, J \subset G$ closed Hence, the sufficiency easily follows Denote $\bar{S}=S_{f, v}$ Let $A \in \ell, e \in E$ Then

$$
e\left(A \times A^{c}\right)=0 \text { and } e\left(A^{c} \times A\right)=0
$$

The definition of $e$ implies

$$
\begin{aligned}
& \int_{X \times G / H_{1}} E\left(A \mid H_{1}\right) E\left(A^{c} \mid H_{2}\right) \bar{S} d \tilde{\mu}_{H_{1}}=0, \\
& \int_{X \times G / H_{1}} E\left(A^{c} \mid H_{1}\right) \cdot E\left(A \mid H_{2}\right) \bar{S} d \tilde{\mu}_{H_{1}}=0
\end{aligned}
$$

Assume that $E\left(A \mid H_{1}\right)\left(x, g H_{1}\right) \neq 0,1$ Hence, by (18) $E\left(A^{c} \mid H_{2}\right) \circ \bar{S}\left(x, g H_{1}\right)=0$, 1.e $E\left(A \mid H_{2}\right) \circ \bar{S}\left(x, g H_{1}\right)=1$ It follows that $\bar{S}\left(x, g H_{1}\right) \subset A$ But, from (19) $E\left(A \mid H_{2}\right) \circ \bar{S}\left(x, g H_{1}\right)=0$ (since $\left.E\left(A^{c} \mid H_{1}\right)\left(x, g H_{1}\right) \neq 0,1\right)$, a contradiction We conclude that for $\tilde{\mu}_{H_{1}}$-a.e $\left(x, g H_{1}\right), E\left(A \mid H_{1}\right)\left(x, g H_{1}\right)=0$ or 1,1 e $A \in \tilde{\mathscr{B}}_{H_{1}}$ Suppose that $A \notin \tilde{\mathscr{B}}_{H_{2}}$ Then for a set of positive $\tilde{\mu}_{H_{1}}$ measure

$$
E\left(A \mid H_{2}\right) \circ \bar{S}\left(x, g H_{1}\right) \neq 0,1
$$

and

$$
E\left(A^{c} \mid H_{2}\right) \circ \bar{S}\left(x, g H_{2}\right) \neq 0,1
$$


However this implies (see (18), (19)) that either $\left(x, g H_{1}\right) \subset A$ or $\left(x, g H_{1}\right) \subset A^{c}$ which is a contradiction Therefore, from (17), $A \in \tilde{\mathscr{B}}_{\mathrm{H}_{1} \mathrm{H}_{2}}$ Moreover

$$
0=\int_{X \times G / H_{1}} \chi_{A} \quad \chi_{A^{c}} \circ \bar{S} d \tilde{\mu}=\int_{X \times G / H_{1}} \chi_{A \cap \bar{S}^{-1} A^{c} d \tilde{\mu}}
$$

forces $\bar{S}^{-1} A=A$ to hold This completes the proof

Let $H$ be the largest closed subgroup of $G$ such that

$$
\ell \subset \tilde{\mathscr{B}}_{H}
$$

Such a group exists, as we can take $H$ as being the closure of the group generated by $\left\{H_{1} \ell \subset \tilde{\mathscr{B}}_{H_{1}}\right\}$ Since the map $\tilde{f} G \rightarrow L^{2}(X \times G, \tilde{\mu})$ given by $\tilde{f}(g)=f \circ g$ where $f \circ g(x, h)=f(x, h g), f \in L^{2}(X \times G, \tilde{\mu})$ is continuous, $\ell \subset \tilde{\mathscr{B}}_{H}$ In other words, there exists a smallest natural factor of $T_{\varphi}$, containıng $\ell$ We will consider this factor as a group extension for which $\ell$ is a factor

LemMa 16 If $H(\ell)=\left\{\bar{S} \in C\left(T_{\varphi}, X \times G / H\right)\right.$ for each $\left.A \in \ell \bar{S}^{-1} A=A\right\}$ then $H(\ell) \subset$ $C_{1}\left(T_{\varphi}, X \times G / H\right)$ (ie all elements from the centralizer of $T_{\varphi}(X \times G / H, \tilde{\mu}) \rightarrow$ $(X \times G / H, \tilde{\mu})$ which do not move any $A \in \ell$ are invertıble $)$

Proof If $\bar{S} \in H(\ell)$ is not invertible, so $\bar{S}^{-1}$ carries the whole $\sigma$-algebra $\tilde{\mathscr{B}}_{H}$ to a smaller sub- $\sigma$-algebra which is a natural factor of $T_{\varphi}(X \times G / H, \tilde{\mu}) \rightarrow$ $(X \times G / H, \tilde{\mu})$ Hence, this factor is determined by a closed (nontrivial) subgroup $F$ of $G / H$ Then it is clear that $\ell \subset \tilde{\mathscr{B}}_{F_{1}}$ where $F_{1}$ is the inverse image of $F$ under the natural map $G \rightarrow G / H$ If $F$ is not trivial, $F_{1} \nsupseteq H$ and we get a contradiction

By exactly the same arguments we can prove the following

Lemma 17 For each e $E$ E (E considered for $\left.T_{\varphi}, T_{\varphi}(X \times G / H, \tilde{\mu}) \rightarrow(X \times G / H, \tilde{\mu})\right)$, $e=(\tilde{\mu})_{\bar{S}}$ and $\bar{S}$ is an invertible element of the centralizer of $T_{\varphi}, T_{\varphi}(X \times G / H, \tilde{\mu}) \rightarrow$ $(X \times G / H, \tilde{\mu})$

THEOREM 3 (Veech Theorem) If $\ell$ is a $T_{\varphi}$-invanant sub- $\sigma$-algebra for an ergodic group extension $T_{\varphi}(X \times G, \tilde{\mu}) \rightarrow(X \times G, \tilde{\mu})$ of a transformation with discrete spectrum, then there exists a natural factor of $T_{\varphi}, T_{\varphi}\left(X \times G / H, \tilde{\mu}_{H}\right) \rightarrow(X \times G / H, \tilde{\mu})$ such that $\ell=\left\{A \in \tilde{\mathscr{B}}_{H}\right.$ for each $\left.\bar{S} \in H(\ell), \bar{S} A=A\right\}$ and $H(\ell)$ is a compact subgroup of the centralizer of $T_{\varphi}(X \times G / H, \tilde{\mu}) \rightarrow(X \times G / H, \tilde{\mu})$

Proof This natural factor is taken as the smallest natural factor of $T_{\varphi}$ which contains $\ell$ Then Lemmata 16 and 17 reduce our problem to the following for this natural factor the relatively independent extension of the diagonal measure on $\ell$ has the ergodic decomposition which consists of some invertible $\bar{S}$ 's belonging to the centralizer of $T_{\varphi}(X \times G / H, \tilde{\mu}) \rightarrow(X \times G / H, \tilde{\mu})$ We are now in the situation of Theorem 182 from [4]

Remark. From Theorem 3 it follows that for each factor $\ell$ of an ergodic group extension $T_{\varphi}(X \times G, \tilde{\mathscr{B}}, \tilde{\mu}) \rightarrow(X \times G, \tilde{\mathscr{B}}, \tilde{\mu})$ we can pass from $\ell$ to $\tilde{\mathscr{B}}$ in two steps, each one of which is a group extension operation (the first not necessarily abelian)

Remark. Although, throughout the paper we have dealt with a discrete spectrum rotation $T(X, \mathscr{B}, \mu) \rightarrow(X, \mathscr{B}, \mu)$, Theorem 3 s still valid if we replace $T$ by a 2 -fold 
simple transformation (see [4]), 1 e a transformation where besides graph joinings $\mu_{S}, S \in C(T)$ we admit only $\mu \times \mu$ as a new ergodic self-joınıng of $T$

Example 1 Let $T(X, \mathscr{B}, \mu) \rightarrow(X, \mathscr{B}, \mu)$ be defined as $T x=x+\alpha$, where $X=[0,1)$ $(\bmod 1), \mu$ is the Lebesgue measure and $\alpha$ is irrational Let $\varphi X \rightarrow X, \varphi(x)=x$ Using the following classical result $([1])$

For $m \in \mathbb{Z}, m \neq 0, b \in[0,1)$ the cocycle

$$
\psi(x)=m x+b \quad \text { is ergodic, }
$$

one can easily compute the centralizer of $T_{\varphi}$ as well as its natural factors $T_{m \varphi}(m \in \mathbb{N})$

$$
\begin{gathered}
C\left(T_{m \varphi}\right)=\left\{S_{f, v}\right\}, \\
f(T x)-f(x)=m \varphi(S x)-v \varphi(x), \\
f(x+\alpha)-f(x)=m(x+\beta)-s m x=m(1-s) x+m \beta
\end{gathered}
$$

Hence, from (20) $s=1$ and using Anzai's result [1], $m \beta=m^{\prime} \alpha$ for an integer $m^{\prime}$ Therefore the centralizer $T_{\varphi}$ does not contain nontrivial compact subgroups, $1 \mathrm{e}$ subgroups for which the projection on the first coordinate is different from $\{1 \mathrm{~d}\}$ Consequently from $C\left(T_{\varphi}\right)$ we can read merely all natural factors, while for instance the transformation

$$
U(x, y)=(x+2 \alpha, x+y)
$$

is a factor of $T_{\varphi}$ (via the map $(x, y) \mapsto(2 x, 2 y)$ ) However this factor can be read from the centralizer of $T_{2 \varphi}$ as the group $\left\{0, \frac{1}{2}\right\}$ can be lifted to the centralizer of $T_{2 \varphi}$

Remark. These circle extensions of some rotations are well-known to be coalescent (1 e their centralizers are groups) However in [6] some new examples of ergodic circle extensions of rotations are constructed with the coalescence property being lost

Example 2 It would be interestıng to know whether for ergodic group extensions the following formula holds

$$
C\left(\left(T_{\varphi}\right)^{n}\right)=C\left(T_{\varphi}\right), \quad n \geq 2
$$

It is not difficult to see that total ergodicity $(1 \mathrm{e}$

$$
\left.\lambda \in J^{e}\left(\left(T_{\varphi}\right)^{n},\left(T_{\varphi}\right)^{n}\right) \text { for each natural } n\right)
$$

of all $\lambda \in J^{e}\left(T_{\varphi}, T_{\varphi}\right)$ forces (21) to be true Indeed, let $\bar{S} \in C\left(\left(T_{\varphi}\right)^{n}\right)$ Then take

$$
\lambda=\frac{1}{n}\left(\tilde{\mu}_{\bar{S}}+\tilde{\mu}_{\bar{S}} \circ T_{\varphi}+\quad+\tilde{\mu}_{\bar{S}} \circ\left(T_{\varphi}\right)^{n-1}\right)
$$

It is not hard to see that $\lambda \in J\left(T_{\varphi}, T_{\varphi}\right)$ is in fact ergodic Then from (22) it follows that $\lambda \in J^{e}\left(\left(T_{\varphi}\right)^{n},\left(T_{\varphi}\right)^{n}\right)$ and consequently $\lambda=\tilde{\mu}_{\bar{S}}$, 1 e $\bar{S} \in C\left(T_{\varphi}\right)$ Nevertheless (21) does not hold in general For instance for the examples from Example 1, $T_{\varphi}(x, y)=(x+\alpha, x+y), C\left(T_{\varphi}\right) \neq C\left(\left(T_{\varphi}\right)^{2}\right)$ as $\frac{1}{2}$ can be lifted to the centralizer of $\left(T_{\varphi}\right)^{2}(x, y)=(x+2 \alpha, 2 x+\alpha+y)$

It is also interesting to ask whether there is any relation between two isomorphic sub- $\sigma$-algebras $\ell_{1}, \ell_{2}$ of a $G$-extension $T_{\varphi}$ and the subgroups $H\left(\ell_{1}\right), H\left(\ell_{2}\right)$ in the centralizers of the smallest natural factors containıng these two sub- $\sigma$-algebras It will follow from Theorem 4 that the answer is positive and the corollary after this theorem says what this relation is 
Assume that $U$ is an 1somorphism of two invariant sub- $\sigma$-algebras $\ell_{1}, \ell_{2}$ of $T_{\varphi}$ Let $X \times G / H_{1}$ and $X \times G / H_{2}$ be the smallest natural factors of $T_{\varphi}$ containing algebras $\ell_{1}$ and $\ell_{2}$, respectively, as factors

THEOREM 4 There exists an isomorphism $\bar{S} X \times G / H_{1} \rightarrow X \times G / H_{2}$ satisfying $\left.\overline{\boldsymbol{S}}\right|_{e_{\mathrm{t}}}=U$

Proof The proof consists of two steps First we will establish the following property of ergodic joinings of $\ell_{1}$ and $\ell_{2}$

If $\nu$ is an ergodic joining of $\ell_{1}$ and $\ell_{2}$ then $\nu$

is the projection of some ergodic joining of $X \times G / H_{1}$ and $X \times G / H_{2}$

Indeed, set $\hat{\nu}$ to be the relatively independent extension of $\nu$ to $\left(X \times G / H_{1}\right) \times$ $\left(X \times G / H_{2}\right), 1 \mathrm{e}$

$$
\hat{\nu}=\int_{\ell_{1} \otimes \ell_{2}} E\left(\mid \ell_{1}\right)(\bar{x}) E\left(\mid \ell_{2}\right)(\bar{y}) d \nu
$$

Obviously, $\hat{\nu}$ need not be ergodic Let

$$
\hat{\nu}=\int_{J^{e}\left(H_{1} H_{2}\right)} \tau d \gamma(\tau)
$$

be the ergodic decomposition of $\hat{v}$

If $\Pi, 1$ the projection of $X \times G / H$, onto the (quotient) Lebesgue space corresponding to $\ell_{1}, l=1,2$, then

$$
\nu=\hat{\nu} \circ\left(\Pi_{1} \times \Pi_{2}\right)=\int_{J^{\mathrm{e}}\left(H_{1}, H_{2}\right)} \tau \circ\left(\Pi_{1} \times \Pi_{2}\right) d \gamma(\tau)
$$

Ergodicity of $\nu$ yields that for $\gamma$-a e $\tau, \tau \circ\left(\Pi_{1} \times \Pi_{2}\right)=\nu$ In particular, there exists an ergodic joining $\tau$ such that $\tau \circ\left(\Pi_{1} \times \Pi_{2}\right)=\nu$, and property (23) is proved

To end the proof of Theorem 4 , denote by $\tilde{\mu}_{U}$ the graph joining on $\ell_{1} \otimes \ell_{2}$, corresponding to the isomorphism $U$ By virtue of (23) there is a measure $\tau \in J^{e}\left(H_{1}, H_{2}\right)$ such that

$$
\tilde{\mu}_{U}=\tau \circ\left(\Pi_{1} \times \Pi_{2}\right)
$$

Take $A \in \ell_{1}$ Then, by definition of $\tilde{\mu}_{U}$,

$$
\tilde{\mu}_{U}\left(A \times U\left(A^{c}\right)\right)=\tilde{\mu}\left(A \cap U^{-1} U\left(A^{c}\right)\right)=0
$$

On the other hand, using (24) we have

$$
\tilde{\mu}_{U}\left(A \times U\left(A^{c}\right)\right)=\tau \circ\left(\Pi_{1} \times \Pi_{2}\right)\left(A \times U\left(A^{c}\right)\right)=\tau\left(A \times U\left(A^{c}\right)\right)
$$

since $A \times U\left(A^{c}\right) \in \ell_{1} \otimes \ell_{2}$

There are subgroups $\tilde{F}_{1} \subset G / H_{1}, \quad l=1,2$, and isomorphism $\bar{S} X \times G / F_{1} \rightarrow$ $X \times G / F_{2}$ (where $F_{1}$ is the subgroup of $G$, for which $G / F_{1}$ is naturally isomorphic to $\left.\left(G / H_{1}\right) / \tilde{F}_{1}, \imath=1,2\right)$ satisfying

$$
\tau=\int_{X \times G / F_{i}} E\left(\mid F_{1}\right) E\left(\mid F_{2}\right) \circ \bar{S} d \tilde{\mu}
$$


Therefore, by (24) and (25)

$$
\begin{aligned}
0 & =\int_{X \times G / F_{1}} E\left(A \mid F_{1}\right)(\bar{x}) E\left(U\left(A^{c}\right) \mid F_{2}\right) \circ \bar{S} d \tilde{\mu} \\
& =\int_{X \times G / F_{1}} E\left(A \mid F_{1}\right)(\bar{x}) E\left(\bar{S}^{-1} U\left(A^{c}\right) \mid F_{1}\right)(\bar{x}) d \tilde{\mu}
\end{aligned}
$$

Simılarly

$$
\begin{aligned}
0 & =\tilde{\mu}_{U}\left(A^{c} \times U(A)\right) \\
& =\int_{X \times G / F_{1}} E\left(A^{c} \mid F_{1}\right)(\bar{x}) \quad E\left(\bar{S}^{-1} U(A) \mid F_{1}\right)(\bar{x}) d \tilde{\mu}
\end{aligned}
$$

Now, if $\tilde{F}_{1}$ is a nontrivial subgroup of $X / H_{1}, 1$ e $F_{1} \nsupseteq H_{1}$, then for some set $A \in \ell_{1}$ the function $E\left(A \mid F_{1}\right)$ is not a characteristic function In other words, for a set of positive measure, $E\left(A \mid F_{1}\right)(\bar{x}) \neq 0,1$ By (26) and (27), for such an $\bar{x}$,

and

$$
0=E\left(A \mid F_{1}\right)(\bar{x}) \quad E\left(\bar{S}^{-1} U\left(A^{c}\right) \mid F_{2}\right)(\bar{x})
$$

$$
0=E\left(A^{c} \mid F_{1}\right)(\bar{x}) \quad E\left(\bar{S}^{-1} U(A) \mid F_{2}\right)(\bar{x})
$$

Using the same arguments as in the proof of Lemma 15, we obtain a contradiction Therefore $F_{1}=H_{1}$ Since $U$ is an isomorphism, $F_{2}=H_{2}$ Thus $\bar{S}$ is an isomorphism of $X \times G / H_{1}$ and $X \times G / H_{2}$ and $\left.\bar{S}\right|_{e_{1}}=U$

COROLLARY 2 If $\ell_{1}, \ell_{2}$ are two isomorphic invariant sub-o-algebras then there is an isomorphism $\bar{S}$ of the smallest natural factors $X \times G / H_{1}$ and $X \times G / H_{2}$ of $T_{4}$ containing $\ell_{1}, \ell_{2}$ respectively, such that $H\left(\ell_{2}\right)=\bar{S} H\left(\ell_{1}\right) \bar{S}^{-1}$

\section{REFERENCES}

[1] H Anzai Ergodic skew-product transformations on the torus Osaka $J$ Math 3 (1951), 83-99

[2] F Hahn \& W Parry Some characteristic properties of dynamical systems with quasi-discrete spectra Math Syst Th 2 (1968), 179-190

[3] A del Junco \& D Rudolph A rank-one, rıgıd, sımple prıme map Ergod Th Dynam \& Sys 7 (1987), 229-247

[4] A del Junco \& D Rudolph On ergodic actıons whose self-joınıngs are graphs Ergod Th \& Dvnam Sys 7 (1987), 531-557

[5] H B Keynes \& D Newton The structure of ergodic measures for compact group extensions Isr $J$ Math 18(4) (1974), 363-389

[6] M Lemańczyk \& P Liardet Coalescence of Anzaı skew products (to appear)

[7] D Newton On canonical factors of ergodic dynamical systems J London Math Soc 2, 19 (1979), 129-136

[8] W Parry Compact abelian group extensions of discrete dynamical systems $Z$ Wahr Verw Geb 13 (1969), 95-113

[9] D Rudolph An example of measure-preservıng map with mınımal self-joinıngs, and applicatıons $J$ d'Analyse Math 35 (1979), 97-122

[10] V S Varadarajan Geometry of Quantum Theory Vol II, Van Nostrand Reinhold, New York (1970)

[11] W A Veech A critenon for a process to be prime Monatsh Math 94 (1982), 335-341 